\title{
Effects of low dose GM-CSF on microglial inflammatory profiles to diverse pathogen-associated molecular patterns (PAMPs) Nilufer Esen and Tammy Kielian*
}

\author{
Address: Department of Neurobiology and Developmental Sciences, University of Arkansas for Medical Sciences, Little Rock, AR 72205, USA \\ Email: Nilufer Esen - EsenNilufer@uams.edu; Tammy Kielian* - KielianTammyL@uams.edu \\ * Corresponding author
}

Published: 20 March 2007

Journal of Neuroinflammation 2007, 4:10 doi:10.1186/1742-2094-4-10

Received: 20 January 2007

Accepted: 20 March 2007

(C) 2007 Esen and Kielian; licensee BioMed Central Ltd.

This is an Open Access article distributed under the terms of the Creative Commons Attribution License (http://creativecommons.org/licenses/by/2.0), which permits unrestricted use, distribution, and reproduction in any medium, provided the original work is properly cited.

\begin{abstract}
Background: It is well appreciated that obtaining sufficient numbers of primary microglia for in vitro experiments has always been a challenge for scientists studying the biological properties of these cells. Supplementing culture medium with granulocyte-macrophage colony-stimulating factor (GM-CSF) partially alleviates this problem by increasing microglial yield. However, GM-CSF has also been reported to transition microglia into a dendritic cell (DC)-like phenotype and consequently, affect their immune properties.

Methods: Although the concentration of GM-CSF used in our protocol for mouse microglial expansion $(0.5 \mathrm{ng} / \mathrm{ml})$ is at least 10 -fold less compared to doses reported to affect microglial maturation and function $(\geq 5 \mathrm{ng} / \mathrm{ml})$, in this study we compared the responses of microglia derived from mixed glial cultures propagated in the presence/absence of low dose GM-CSF to establish whether this growth factor significantly altered the immune properties of microglia to diverse bacterial stimuli. These stimuli included the gram-positive pathogen Staphylococcus aureus (S. aureus) and its cell wall product peptidoglycan (PGN), a Toll-like receptor 2 (TLR2) agonist; the TLR3 ligand polyinosine-polycytidylic acid (polyl:C), a synthetic mimic of viral double-stranded RNA; lipopolysaccharide (LPS) a TLR4 agonist; and the TLR9 ligand $C_{P G}$ oligonucleotide (CPG-ODN), a synthetic form of bacteria/viral DNA.

Results: Interestingly, the relative numbers of microglia recovered from mixed glial cultures following the initial harvest were not influenced by GM-CSF. However, following the second and third collections of the same mixed cultures, the yield of microglia from GM-CSF-supplemented flasks was increased two-fold. Despite the ability of GM-CSF to expand microglial numbers, cells propagated in the presence/absence of GM-CSF demonstrated roughly equivalent responses following $S$. aureus and PGN stimulation. Specifically, the induction of tumor necrosis factor- $\alpha$ (TNF- $\alpha$ ), macrophage inflammatory protein-2 (MIP-2/CXCL2), and major histocompatibility complex (MHC) class II, CD80, CD86 expression by microglia in response to $S$. aureus were similar regardless of whether cells had been exposed to GM-CSF during the mixed culture period. In addition, microglial phagocytosis of intact bacteria was unaffected by GM-CSF. In contrast, upon $S$. aureus stimulation, CD40 expression was induced more prominently in microglia expanded in GM-CSF. Analysis of microglial responses to additional pathogen-associate molecular patterns (PAMPs) revealed that low dose GM-CSF did not significantly alter TNF- $\alpha$ or MIP-2 production in response to the TLR3 and TLR4 agonists polyl:C or LPS, respectively; however, cells expanded in the presence of GM-CSF produced lower levels of both mediators following CPG-ODN stimulation.
\end{abstract}

Conclusion: We demonstrate that low levels of GM-CSF are sufficient to expand microglial numbers without significantly affecting their immunological responses following activation of TLR2, TLR4 or TLR3 signaling. Therefore, low dose GM-CSF can be considered as a reliable method to achieve higher microglial yields without introducing dramatic activation artifacts. 


\section{Background}

Granulocyte-macrophage colony-stimulating factor (GMCSF) is a well known hematopoietic cytokine produced primarily by $\mathrm{T}$ cells, macrophages, endothelial cells, and fibroblasts [1-4]. GM-CSF was originally defined based on its ability to stimulate the differentiation and function of granulocytes, monocytes and macrophages [1]. In addition, previous studies have established that GM-CSF promotes the survival and proliferation of neonatal rat, mouse, and human microglia in culture [5-9]. Based on these observations, GM-CSF is commonly used as a culture medium supplement to obtain sufficient numbers of microglia to conduct downstream in vitro experiments [10-12]. However, recent studies have suggested that microglia are not terminally differentiated and that GMCSF can induce their functional maturation and expression of dendritic cell (DC) markers [13-15], which has raised concerns with investigators who are examining the immunological functions of primary microglia in various CNS pathologies. For example, GM-CSF has been reported to induce the transcription of genes important for $\mathrm{T}$ cell activation, chemotaxis, antigen processing, innate immunity, and immunosuppression, suggesting the transition of microglia into a more professional antigen presenting cell phenotype [14-16]. In addition, other studies have utilized GM-CSF to induce DC maturation from myeloid progenitor cells $[17,18]$. Overall, these studies suggest that microglia can transition into a DClike phenotype when cultured in the presence of adequate levels of GM-CSF.

During the preparation of our mouse primary mixed glial cultures, we routinely supplement culture medium with low levels of GM-CSF $(0.5 \mathrm{ng} / \mathrm{ml})$ to increase microglial yields. Despite the fact that this concentration is approximately ten-fold lower than what has been reported to modulate microglial function and transition into a DC phenotype (i.e. $5-50 \mathrm{ng} / \mathrm{ml}$ ), we are often questioned regarding the consequences of microglial exposure to GMCSF during the mixed glial culture period and whether this introduces artifacts in the activation profiles of these cells in subsequent in vitro experiments. Therefore, the primary objective of the present study was to evaluate whether low dose GM-CSF leads to alterations in microglial morphology and/or functional activation in response to a wide variety of PAMPs commonly associated with various CNS infections, namely Staphylococcus aureus (S. aureus) and its cell wall component peptidoglycan (PGN), LPS, polyI:C, and CpG-ODN. Our results demonstrate that low dose GM-CSF led to a significant expansion in microglial numbers without affecting their phagocytic activity or cytokine production profiles in response to the majority of PAMPs examined, with the exception of the TLR9 agonist CpG-ODN. However, we did observe a phenotypic transformation of microglia expanded in the pres- ence of low dose GM-CSF, namely a transition to a DClike morphology typified by numerous dendrites; however, the functional implication(s) of this change remain to be determined. Therefore, low dose GM-CSF can be successfully utilized as a culture medium supplement to enhance microglial recovery without overtly compromising normal responsiveness to microbial stimuli. Importantly, these findings exclude any potential GM-CSFinduced artifacts in the read-outs of microglial activation routinely used in our studies.

\section{Methods}

\section{Primary microglia cell culture and reagents}

Primary microglia were isolated from inbred neonatal C57BL/6 mice as previously described [19]. Briefly, agematched litters (postnatal day 2-5) were euthanized using an overdose of inhaled Halothane (Halocarbon Laboratories, River Edge, NJ) to obtain mixed glial cultures. Cerebri were collected under aseptic conditions and the meninges removed. Tissues were minced, resuspended in trypsinEDTA (Mediatech Inc., Herndon, VA), and incubated at $37^{\circ} \mathrm{C}$ for 20 minutes. Subsequently, cells were resuspended in complete DMEM ( $4.5 \mathrm{~g} / \mathrm{L}$ glucose, Mediatech Inc.) containing 10\% FBS (Hyclone, Logan, UT), $200 \mathrm{mM}$ L-glutamine, $100 \mathrm{U} / \mathrm{ml}$ penicillin, $0.1 \mathrm{mg} / \mathrm{ml}$ streptomycin and $0.25 \mu \mathrm{g} / \mathrm{ml}$ fungizone (all from Mediatech Inc.), OPI supplement (oxalacetic acid, pyruvate, insulin; Sigma, St. Louis, MO), and $0.5 \mathrm{ng} / \mathrm{ml}$ recombinant mouse GM-CSF (BD Pharmingen, San Diego, CA). The cell suspension was further triturated and filtered through a 70 $\mu \mathrm{m}$ cell strainer. Subsequently, cells were centrifuged, resuspended in complete medium, and seeded into 150 $\mathrm{cm}^{2}$ flasks. To minimize variation between microglia expanded in the presence/absence of GM-CSF, mouse pups were procured from litters born on the same day and primary cultures propagated with or without GM-CSF were derived from the same initial mixed glial population (i.e. half of the mixed glial cells recovered were cultured with GM-CSF $(+)$ medium while the other half was cultured without GM-CSF).

Upon confluence (7-10 days), flasks were shaken overnight at $200 \mathrm{rpm}$ at $37^{\circ} \mathrm{C}$ to recover microglia. Microglia from both (+) GM-CSF- and (-) GM-CSF-treated flasks were collected and plated in medium without GM-CSF for all subsequent experiments. The purity of microglial cultures was evaluated by immunohistochemical staining using antibodies against CD11b and GFAP to identify microglia and astrocytes, respectively, and was routinely greater than $95 \%$. Each experiment presented in this paper was initially performed with microglia collected after the first shake and repeated with cells collected after a second shake to confirm that the responses were comparable. Based on our findings that microglial responses were similarly affected in all experiments regardless of when they 
were harvested, we concluded that microglial responsiveness to microbial stimuli does not significantly differ in cells collected from the first versus subsequent shakes.

Heat-inactivated $S$. aureus (strain RN6390) was prepared as previously described [11] and PGN derived from $S$. aureus, poly I:C, and the synthetic CpG oligonucleotide ODN1826 were obtained from InvivoGen (San Diego, CA). Escherichia coli O11:B1 LPS was purchased from List Biological Laboratories (Campbell, CA). The doses of stimuli used throughout this report were based on our previous studies that established optimal cytokine responses induced following bacterial stimulation without any evidence of toxicity $[20,21]$. All non-LPS reagents were verified to have endotoxin levels $<0.03 \mathrm{EU} / \mathrm{ml}$ as determined by Limulus amebocyte lysate assay (Associates of Cape Cod, Falmouth, MA).

\section{Enzyme linked immunosorbent assay (ELISA)}

Protein levels of TNF- $\alpha$, IL-12p40 (OptEIA, BD Pharmingen) and macrophage inflammatory protein (MIP-2/ CXCL2, DuoSet, R\&D Systems, Minneapolis, MN) were quantified in conditioned medium from PAMP-stimulated microglia using ELISA kits according to the manufacturer's instructions (level of sensitivity $=15.6 \mathrm{pg} / \mathrm{ml}$ ).

\section{Cell viability assays}

To evaluate whether microglia expanded in the presence or absence of GM-CSF demonstrated similar survival profiles following bacterial activation, a standard MTT assay based upon the mitochondrial conversion of (3- [4,5dimethylthiazol-2-yl]-2,5-diphenyl-tetrazolium bromide (MTT) into formazan crystals was performed as previously described [22].

\section{Phagocytosis assay}

Primary microglia expanded with or without GM-CSF were seeded onto $12 \mathrm{~mm}$ coverslips in 24-well plates and incubated overnight. The following day, cells were treated with a heat-killed $S$. aureus isolate that constitutively expresses green fluorescence protein (GFP, kindly provided by Dr. Ambrose Cheung, Dartmouth Medical School) for $3 \mathrm{~h}$, whereupon Hoechst 33342 (Molecular Probes, Eugene, OR) was added to visualize nuclei. Cells were washed extensively with PBS and incubated with $0.05 \%$ crystal violet in $0.15 \mathrm{M} \mathrm{NaCl}$ for 45 seconds to quench any fluorescence emitted by residual extracellular bacteria. Coverslips were viewed under fluorescence microscopy using an excitation wavelength of $460-490$ nm (FITC filter, Olympus BX41, Tokyo, Japan).

\section{Immunofluorescence staining and confocal microscopy}

Primary microglia expanded in the presence/absence of GM-CSF were seeded onto $12 \mathrm{~mm}$ coverslips in 24-well plates and incubated overnight. The following day, micro- glia were treated with $10^{7}$ heat-inactivated $S$. aureus for 24 $\mathrm{h}$, whereupon cells were washed extensively with PBS, fixed in ice-cold methanol, and incubated with PBS $/ 10 \%$ donkey serum to prevent non-specific antibody binding (Jackson ImmunoResearch, West Grove, PA) for $30 \mathrm{~min}$ at room temperature. Subsequently, microglia were incubated with a MHC class-II antibody (rat anti-mouse, BD Pharmingen) overnight at $4{ }^{\circ} \mathrm{C}$. The following day, cells were incubated with a donkey anti-rat biotinylated secondary antibody (Vector Laboratories, Burlingame, CA) and detected using a streptavidin-Alexa Fluor 568 conjugate (Molecular Probes). Subsequently, a directly conjugated CD11b-FITC antibody (BD Pharmingen) was added and cells incubated for $1 \mathrm{~h}$ at $37^{\circ} \mathrm{C}$, whereupon Hoechst 33342 was used to visualize nuclei. Controls included microglia incubated with secondary antibodies only to assess the extent of non-specific staining. Coverslips were imaged using a Zeiss laser scanning confocal microscope (LSM 510, Carl Zeiss Microimaging). Hoechst 33342 for nuclear visualization was excited by a $405 \mathrm{~nm}$ diode laser, FITC to visualize CD11b immunoreactivity was excited with a $488 \mathrm{~nm}$ argon laser, and Alexa Fluor 568 to demonstrate MHC class II expression was excited with a 561 nm DPSS laser, with images collected using the appropriate emissions. The confocal pinhole was set to obtain an optical section thickness of $1.6 \mu \mathrm{m}$. To demonstrate colocalization of CD11b, MHC class II, and Hoechst 33342 signals, RGB merges of individual confocal images were performed using the ImageJ software program (NIH Image).

\section{Flow cytometry}

Primary microglia expanded in the presence/absence of GM-CSF were seeded into 6 -well plates $\left(2 \times 10^{6}\right.$ cells/ well), and incubated overnight. The following day, cells were treated with $10^{7}$ heat-inactivated $S$. aureus for $24 \mathrm{~h}$. At the end of the incubation period, cells were washed twice with PBS, and collected using a cell scraper. A total of $5 \times 10^{5}$ microglia in each group were stained for twocolor flow cytometry using CD11b-FITC and CD11c-PECy7 antibodies (both from BD Pharmingen). Fc receptors were blocked with the addition of Fc block ${ }^{\mathrm{TM}}$ (anti-CD16 and -CD32 cocktail, BD Pharmingen). In addition, a separate set of microglia were stained with antibodies directed against the co-stimulatory molecules CD40, CD80, and CD86, in addition to MHC class II (all from BD Pharmingen), and subsequently incubated with a donkey anti-rat IgG FITC-conjugated secondary antibody (Jackson ImmunoResearch Laboratories, West Grove, PA). Controls included microglia incubated with appropriate isotype control-matched antibodies to assess the extent of non-specific background staining. Cells were analyzed using a FACS Calibur cytometer (BD Biosciences, San Jose, CA) with settings based on the staining of microglia with isotype control antibodies alone. 


\section{Morphological analysis}

Primary microglia propagated in the presence/absence of GM-CSF were seeded into $35 \mathrm{~mm}$ dishes $\left(2 \times 10^{6}\right.$ cells/ well), and incubated overnight. The following day, cells were treated with $10^{7}$ heat-inactivated $S$. aureus for $24 \mathrm{~h}$ or left unstimulated. At the end of the incubation period, cell morphology was visualized by phase-contrast microscopy and images collected using a fixed stage upright epifluorescence microscope (BX51WI, Olympus) equipped with a $40 \times$ water immersion objective lens and a 12-bit intensified monochrome CCD camera (CoolSnap ES, Photometrics, Tucson, AZ).

\section{Statistics}

Significant differences between experimental groups were determined by the $t$-test for unequal variances at the 95\% confidence interval using Sigma Stat (SPSS Science, Chicago, IL).

\section{Results}

\section{Microglial recovery is enhanced following low dose GM- CSF treatment}

It has been well established that GM-CSF exerts mitogenic effects on primary microglia, effectively expanding cell numbers $[5,6,10,12,23]$. Therefore, the use of this cytokine during the mixed glial culture period reduces the number of neonatal mice required to obtain sufficient numbers of microglia for subsequent studies. We routinely supplement our culture medium with a relatively low dose of GM-CSF; however, to date, we have not yet performed a detailed analysis regarding the efficacy of this dose on microglial expansion or more importantly, whether GM-CSF alters the subsequent responsiveness of microglia compared to cells that have never been exposed to exogenous growth factor. This became an essential issue to address since we are often questioned about the consequences of GM-CSF treatment on downstream microglial responses, thus forming the impetus for the current study. Work by others had demonstrated that GMCSF led to the transition of microglia into macrophage and/or dendritic-like cells [13-15,18]; however, the doses of cytokine used to drive this differentiation (i.e. 10-50 $\mathrm{ng} / \mathrm{ml}$ ) ranged anywhere from 20- to 50-fold higher than the concentration used to expand microglia numbers in our experiments (i.e. $0.5 \mathrm{ng} / \mathrm{ml}$ ). In addition, other important factors to consider include whether microglia are procured from neonatal versus adult animals or if microglia are expanded as mixed glial cultures or as purified cells.

To initiate our analysis of low dose GM-CSF effects on microglia, we quantified the relative percentages of microglia recovered from mixed glial cultures continuously propagated in the presence/absence of recombinant mouse GM-CSF $(0.5 \mathrm{ng} / \mathrm{ml})$. Upon reaching confluence, mixed glial cultures were collected at three consecutive harvests separated by an interval of 7-10 days. The number of microglia collected after the first harvest was not significantly influenced by GM-CSF; however, after the second and third harvests the number of microglia collected from GM-CSF-negative flasks was significantly lower compared to those supplemented with GM-CSF (Figure 1 and data not shown). This finding demonstrates that even at very low levels, GM-CSF is still capable of expanding microglial numbers, obviating the need for large numbers of neonatal animals to achieve sufficient cell yields.

\section{Low dose GM-CSF leads to morphological changes in microglia characteristic of dendritic cells (DCs)}

Microglia display an ameboid morphology during embryogenesis [24,25] and assume a ramified shape upon maturation in the normal brain under physiological conditions $[24,26,27]$. However, in response to injury or infection, microglia become activated and transition into an ameboid morphology [27]. In vitro, ameboid microglia are rounded cells flattened to the substratum and have

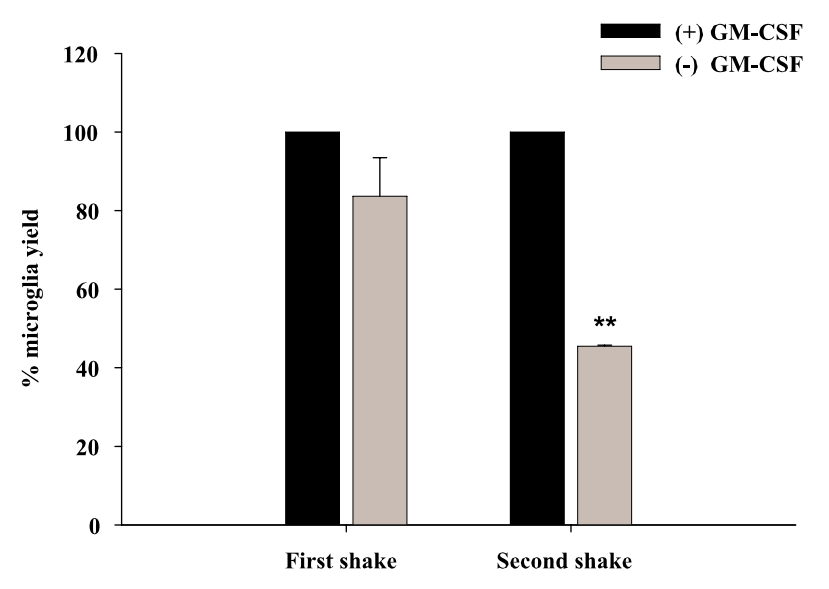

Figure I

GM-CSF improves microglial yields from primary cultures. Mixed glial cells were prepared in culture medium supplemented with (+) or without (-) GM-CSF $(0.5 \mathrm{ng} / \mathrm{ml})$. Upon confluence (7-10 days), flasks were shaken overnight and the following day, supernatants were collected and microglial cell counts performed. Flasks were shaken once a week and following the second shake the relative percentage of microglia recovered from flasks cultured without GM-CSF was significantly reduced $(* *, p<0.00 I)$. For reporting differences in microglial recovery, we normalized (i.e. divided) the numbers of microglia recovered from GM-CSF (-) flasks by those collected from GM-CSF (+) cultures to express the former as a percentage of GM-CSF (+)-containing conditions (set to $100 \%$ ). Results represent the mean \pm SD of two independent experiments. 
been reported to function as phagocytic antigen presenting cells $[28,29,30,31,32]$. This ameboid morphology observed in vitro is likely a consequence of the isolation procedure where, in general, ameboid characteristics are more typical of neonatal microglia, whereas adult cells normally exhibit a more quiescent ramified phenotype [33-36]. Nonetheless, ramification of ameboid microglia can be achieved by either growing cells on an astrocyte monolayer, culturing microglia in astrocyte-conditioned media, or treating with M-CSF $[15,28,33]$.

Although it is well established that GM-CSF induces microglial proliferation $[5,6]$, there are conflicting reports in the literature regarding its effects on microglial morphology. For example, GM-CSF ( $5 \mathrm{U} / \mathrm{ml}$ for $72 \mathrm{~h}$ ) has been shown to increase the number of ameboid microglia by 5 - to 6 -fold [6], whereas another study reported that GM-CSF induced microglial ramification and LPS treatment transformed cells to an ameboid phenotype [37]. To further complicate matters, recent studies have reported that exposure of purified adult microglia to GM-CSF led to their transformation into a macrophage- or DC-like morphology $[13,15,38]$. However, it is important to note that the relative concentrations of GM-CSF used in these studies were relatively high (i.e. $5-50 \mathrm{ng} / \mathrm{ml}$ ) compared to our experiments $(0.5 \mathrm{ng} / \mathrm{ml})$. Therefore, to establish the effect of low dose GM-CSF on microglial morphology we investigated cells derived from our culture conditions by phasecontrast microscopy. As shown in Figure 2, unstimulated microglia expanded in the absence of GM-CSF appeared as single rounded or clustered cells that were relatively small in size. In contrast, microglia propagated in the presence of GM-CSF were more flattened, enlarged, and displayed a sizeable number of dendritic processes reminiscent of DCs (Figure 2) [15,38]. Interestingly, when stimulated with $S$. aureus, microglial morphology was virtually indistinguishable between cells that had been expanded in the presence/absence of GM-CSF (Figure 2). $S$. aureus-activated microglia appeared heterogeneous in shape compared to unstimulated cells typified by the presence of round, elongated, and flattened cells that exhibited the characteristic homotypic adhesion we have observed in our previous studies ([39] and unpublished observations). Collectively, these results indicate that exposure of mixed glial cultures to low dose GM-CSF leads to morphological alterations in purified "resting" microglia that are reminiscent of DCs, in agreement with studies by other groups using high concentrations of GM-CSF $[15,33,38]$. However, the morphological transformation associated with $S$. aureus activation of microglia is similar, regardless of prior exposure to GM-CSF.

\section{Microglial expansion with low dose GM-CSF leads to differential responses to various PAMPs}

It has been suggested that GM-CSF plays an important role in promoting the proinflammatory functions of primary microglia, since higher cytokine doses have been reported to induce the transcription of several proinflammatory mediators in neonatal microglia [14] as well as enhance the antigen presentation properties of adult microglia $[10,14,15,32,40]$. Although our previous studies using primary neonatal mouse microglia expanded in the presence of low dose GM-CSF did not detect significant constitutive proinflammatory mediator expression under resting conditions $[11,20,23,41,42]$, we have not yet performed a detailed side-by-side comparison of activation profiles of microglia propagated in the presence/ absence of GM-CSF during the mixed glial culture period. Therefore, in the present study, we evaluated proinflammatory mediator expression by microglia expanded with or without GM-CSF in response to a diverse array of PAMPs to establish the utility of low dose GM-CSF for microglial expansion without affecting downstream cellular responsiveness. The PAMPs evaluated included the gram-positive bacterium $S$. aureus and its cell wall component PGN, as well as polyI:C, LPS, and CpG-ODN. Similar to our previous results we found that propagating microglia in the presence of GM-CSF did not alter any constitutive proinflammatory mediator production or significantly affect the degree of responsiveness to either $S$. aureus or PGN (Figure 3). Specifically, TNF- $\alpha$, MIP-2, and IL-12 p40 were produced to equivalent extents upon $S$. aureus and PGN activation by microglia expanded in the presence/absence of GM-CSF (Figure 3A and 3B and data not shown). To further examine the effects of low dose GM-CSF on microglial responses, we broadened our analysis to investigate several PAMPs acting through TLRs other than TLR2, in particular, polyI:C, LPS, and CpGODN, which stimulate microglia via TLR3, TLR4, and TLR9, respectively $[12,43,44]$. Of all of the PAMPs examined, responses to CpG-ODN were most affected by whether microglia had been expanded in the presence of low dose GM-CSF. Specifically, both TNF- $\alpha$ and MIP-2 production were significantly reduced in CpG-ODN treated cells that had no prior exposure to GM-CSF (Figure $4 \mathrm{~A}$ and $4 \mathrm{~B}$ ). In contrast, responses to polyI:C and LPS remained unchanged or modestly affected, respectively (Figure 4), suggesting that low dose GM-CSF does not drastically alter microglial responsiveness to these PAMPs, in terms of the proinflammatory mediators examined here. Interestingly, polyI:C was not able to induce MIP-2 production in microglia above baseline levels regardless of GM-CSF exposure (Figure 4A, and 4B). Importantly, the concentrations of all stimuli used in this study did not adversely affect microglial viability, indicating that the exposure of microglia to GM-CSF during the expansion 


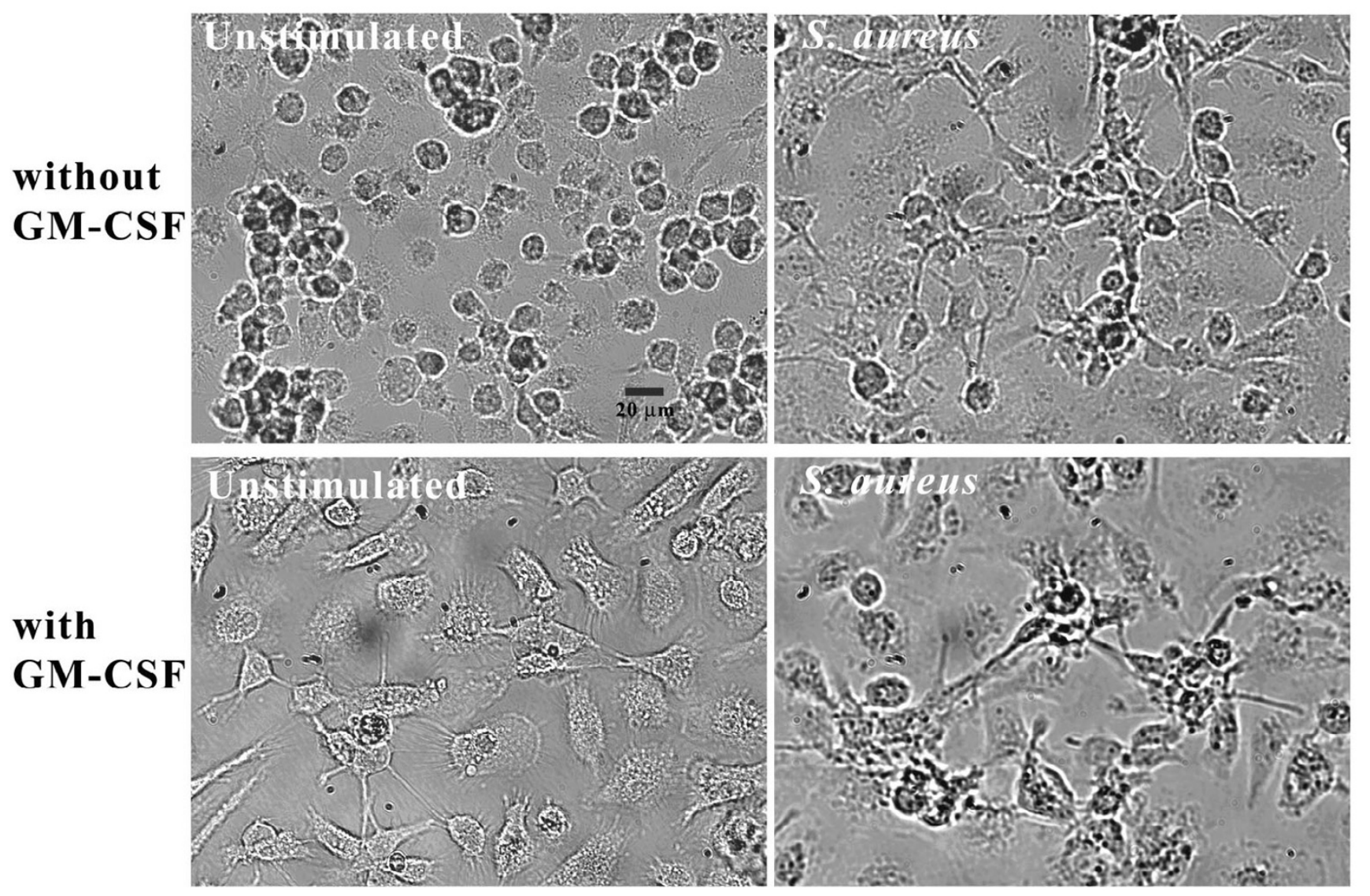

\section{Figure 2}

Exposure of mixed glial cultures to low GM-CSF results in the ramification of resting microglia with a DC-like appearance. Primary microglia expanded either with or without GM-CSF were seeded onto $35-\mathrm{mm}$ dishes at $2 \times 10^{6}$ cells per dish and incubated overnight in 6-well plates. The following day, cells were either unstimulated or treated with heat-inactivated S. aureus ( $10^{7} \mathrm{cfu} /$ well) for $24 \mathrm{~h}$, whereupon bright field phase-contrast images were collected (40x). The results pictured are representative of two independent experiments.

period did not sensitize cells to activation-dependent toxicity (Figures 3C and 4C).

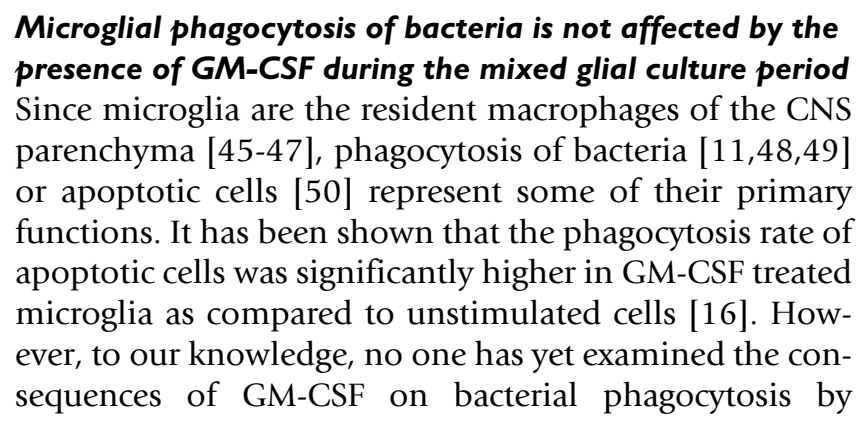

microglia. In the present study we found that the expansion of primary microglia with GM-CSF did not affect their phagocytic activity. As shown in Figure 5A, both microglia cultured with or without GM-CSF were able to engulf GFP-labeled S. aureus to equivalent extents, where the phagocytic index (as determined by quantitating the number of microglia harboring intracellular bacteria) was not significantly different between the groups (Figure 5B). Although distinct phagocytic pathways are likely involved, our findings are similar to those of Fischer et al. (1993) where the percentage of microglia phagocytizing latex beads did not significantly differ following GM-CSF treatment [10]. Collectively, these results suggest that any 
A

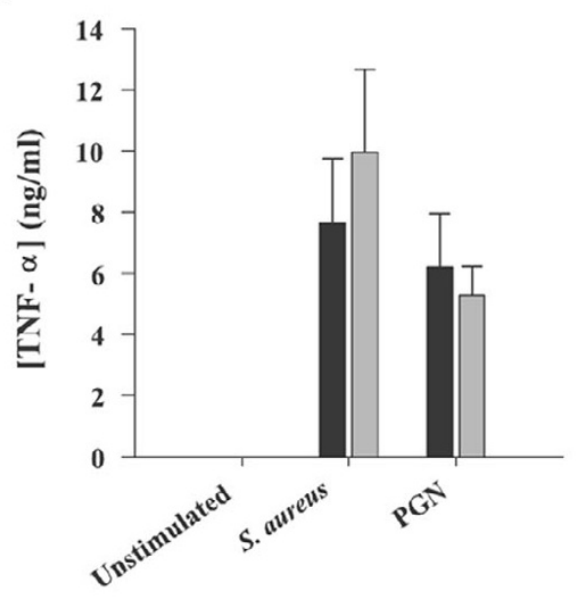

B

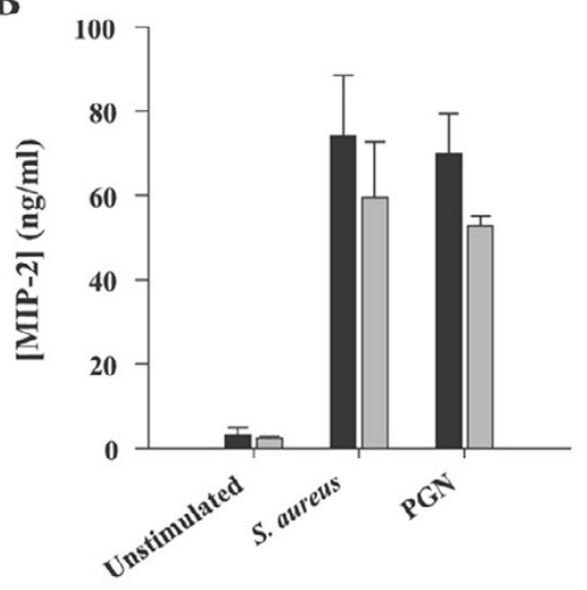

C

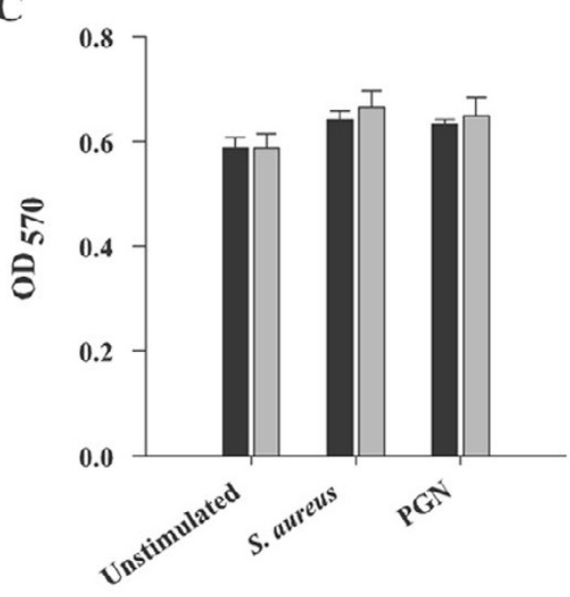

Figure 3

Low dose GM-CSF does not alter microglial cytokine/chemokine responses to $S$. aureus and PGN. Primary microglia expanded with (+) or without (-) GM-CSF were exposed to heat-inactivated S. aureus (107 cfu/well) or PGN (I0 $\mu \mathrm{g} /$ $\mathrm{ml}$ ) for $24 \mathrm{~h}$, whereupon conditioned supernatants were collected and analyzed for TNF- $\alpha$ (A) and MIP-2 (B) expression by ELISA (mean $\pm \mathrm{SD}$ ). Microglial cell viability was assessed using a standard MTT assay and the raw $\mathrm{OD}_{570}$ absorbance values are reported (C). Results are representative of three independent experiments. 


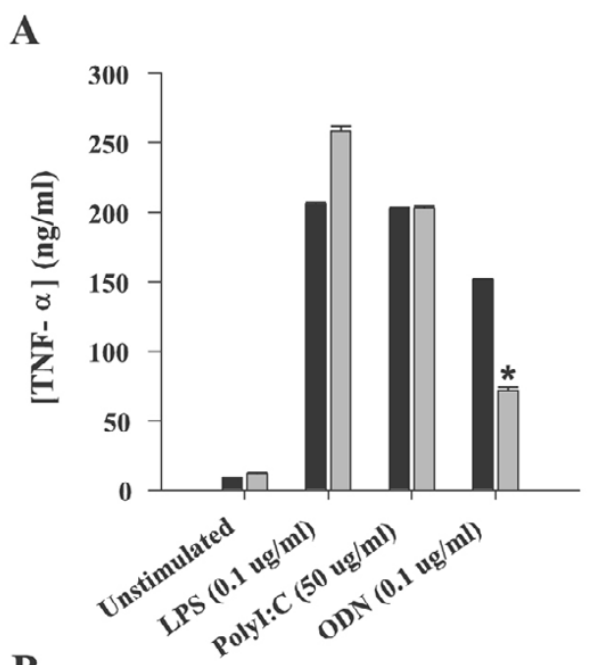

B

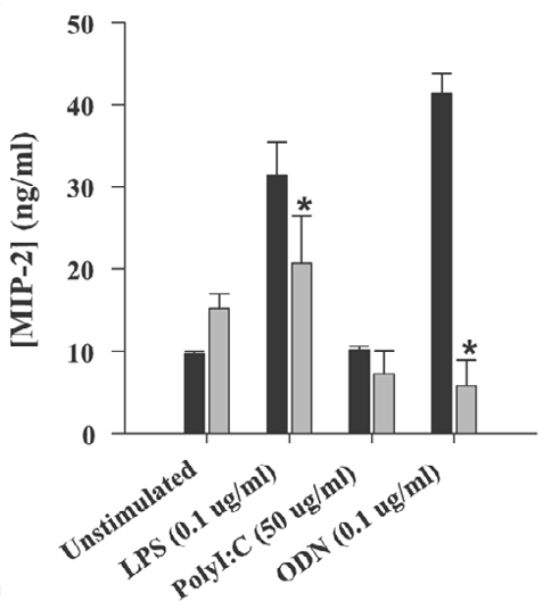

C

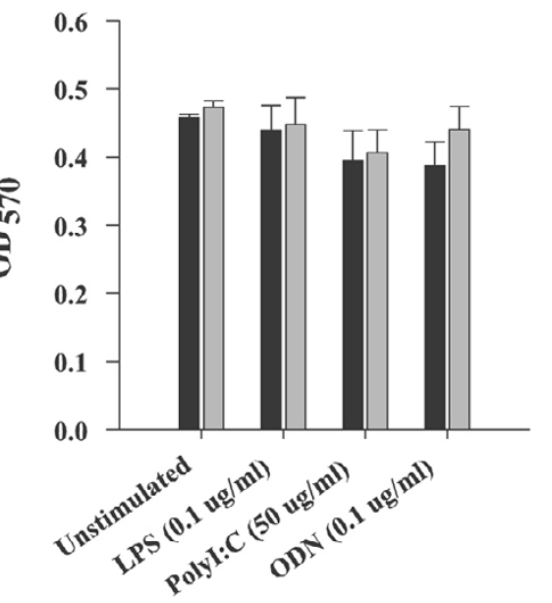

Figure 4

Low dose GM-CSF influences microglial responsiveness to a downstream CpG-ODN (TLR9) stimulus. Primary microglia expanded with (+) or without (-) GM-CSF were exposed to various concentrations of LPS, polyl:C or CpG-ODN for $24 \mathrm{~h}$, whereupon conditioned supernatants were collected and analyzed for TNF- $\alpha$ (A) and MIP-2 (B) expression by ELISA (mean \pm SD). Microglial cell viability was assessed using a standard MTT assay and the raw $O_{570}$ absorbance values are reported (C). Results are representative of three independent experiments. Asterisks denote significant differences between microglia propagated in the presence and absence of GM-CSF $(* p<0.05, * * p<0.001)$. 
effects of GM-CSF on microglial activation and bacterial phagocytosis are negligible at the low doses used as a culture medium supplement in our studies.

\section{Effects of low dose GM-CSF on the expression of microglial surface markers}

Primary microglia can be differentiated from macrophages based on their characteristic staining pattern of CD $11 b^{\text {high }}$ and CD45 low $[32,51-53]$. Although it has been shown that GM-CSF used as either a culture medium supplement or a direct stimulus leads to a dramatic expansion in microglial numbers $[10,13]$, an effect on CD11b expression was not demonstrated [13]. On the other hand, GM-CSF has been reported to inhibit the IFN- $\gamma$ induced expression of another surface marker, MHC class II, and as a consequence, modulate microglial APC functions $[15,54]$. However, Fischer at al. (1993) have shown that MHC class II expression is not altered on microglia grown in the presence of GM-CSF, whereas its expression is induced following IFN- $\gamma$ treatment [10].

In the present study we have demonstrated that constitutive CD11b expression was slightly enhanced in GM-CSFexpanded microglia compared to cells cultured without GM-CSF as determined by both immunofluorescence staining and FACS analysis (Figures 6A and 8, respectively). In addition CD11b expression was moderately increased following $S$. aureus stimulation regardless of whether microglia had been propagated in the presence or absence of GM-CSF (Figure 6A and 6B and Figure 8). With regard to $\mathrm{MHC}$ class II expression, considerable immunoreactivity was observed in "resting" microglia, similar to what has been reported by others with cultured neonatal microglia (Figure 6A) [10,14,54]. This constitutive MHC class II expression was not influenced by GM-CSF during the mixed glial culture period (Figure 6A). Unexpectedly, in contrast to what was observed with CD11b, S. aureus stimulation did not lead to a notable increase in MHC class II immunoreactivity and no obvious modulation by GM-CSF was observed (Figure 6B). This finding was independently confirmed by flow cytometric staining (Figure 7 ). The inability of $S$. aureus to augment MHC class II levels was unexpected, but could be explained by the fact that the constitutive MHC class II levels detected may not be subject to further increases following cell stimulation. Importantly, the degree of non-specific background staining observed was negligible (Figure 6C).

Since neonatal microglia exhibit a partially activated phenotype in vitro, as indicated by an intermediate expression level of co-stimulatory molecules in addition to MHC class II [52,55], we expanded our analysis to evaluate the expression of several co-stimulatory molecules including CD40, CD80 and CD86 by flow cytometry to determine the possible effects of low dose GM-CSF on microglial expression of these molecules. Similar to MHC class II, baseline CD80 and CD86 expression was not influenced by either GM-CSF or S. aureus (Figure 7). On the other hand, microglia propagated in the presence of low dose GM-CSF displayed elevated CD40 levels following S. aureus stimulation (Figure 7), whereas under resting conditions, CD40 levels were equivalent in microglia expanded with or without GM-CSF. Taken together, this data suggests that exposing microglia to low doses of GMCSF during the mixed culture period does not lead to significant alterations in surface marker expression, with the exception of CD40, which may have an impact on microglial activation in the presence of CD40 ligands.

The adult brain parenchyma harbors a population of CD $11 b^{+}$myeloid precursors $[51,56-58]$ that can be driven to differentiate into immature DCs by GM-CSF treatment as measured by the induction of cell surface markers such as DEC-205, CD11c, and CD80 [10,13,38,40]. When Fischer and co-workers (2001) incubated primary microglia from adult mouse brain with GM-CSF (50 ng/ml) for 5 days, approximately $30 \%$ of these cells expressed CD $11 \mathrm{c}$ compared to $<0.5 \%$ of microglia in the initial population. In our culture system, microglia are exposed to GM-CSF for a period of longer than 10 days and this was not sufficient to induce CD11c expression since, when compared to isotype control staining, none of the CD11c signal detected could be attributed to specific binding in either the "resting state" or following $S$. aureus exposure (Figure 8 ). Collectively, these findings indicate that exposure of microglia to low dose GM-CSF during the mixed glial culture period does not induce the expression of the classical DC marker CD11c.

\section{Discussion}

GM-CSF, which is a potent stimulator of microglia as well as macrophages and granulocytes, is usually detected in the brain following $\mathrm{T}$ cell infiltration $[14,59,60]$ or produced by activation of astrocytes and/or endothelial cells $[7,61]$. The amount of GM-CSF released by these cells could be sufficient to induce a proinflammatory state of local microglia and/or their transformation into a DC- or macrophage-like cell. Although numerous studies have been performed examining the effects of GM-CSF on microglial morphology and function, often times these reports have produced conflicting results $[10,14,16,54]$. This likely stems from differences in GM-CSF treatment paradigms, species of microglial origin, and/or whether adult or neonatal cells are used. All of these issues coupled with the fact that the doses of GM-CSF applied to microglia have shown tremendous variability among individual studies, makes it quite difficult to compare results and arrive at general conclusions regarding the effects of GMCSF on primary microglia. When we reviewed the literature we found that GM-CSF was mainly evaluated in two 

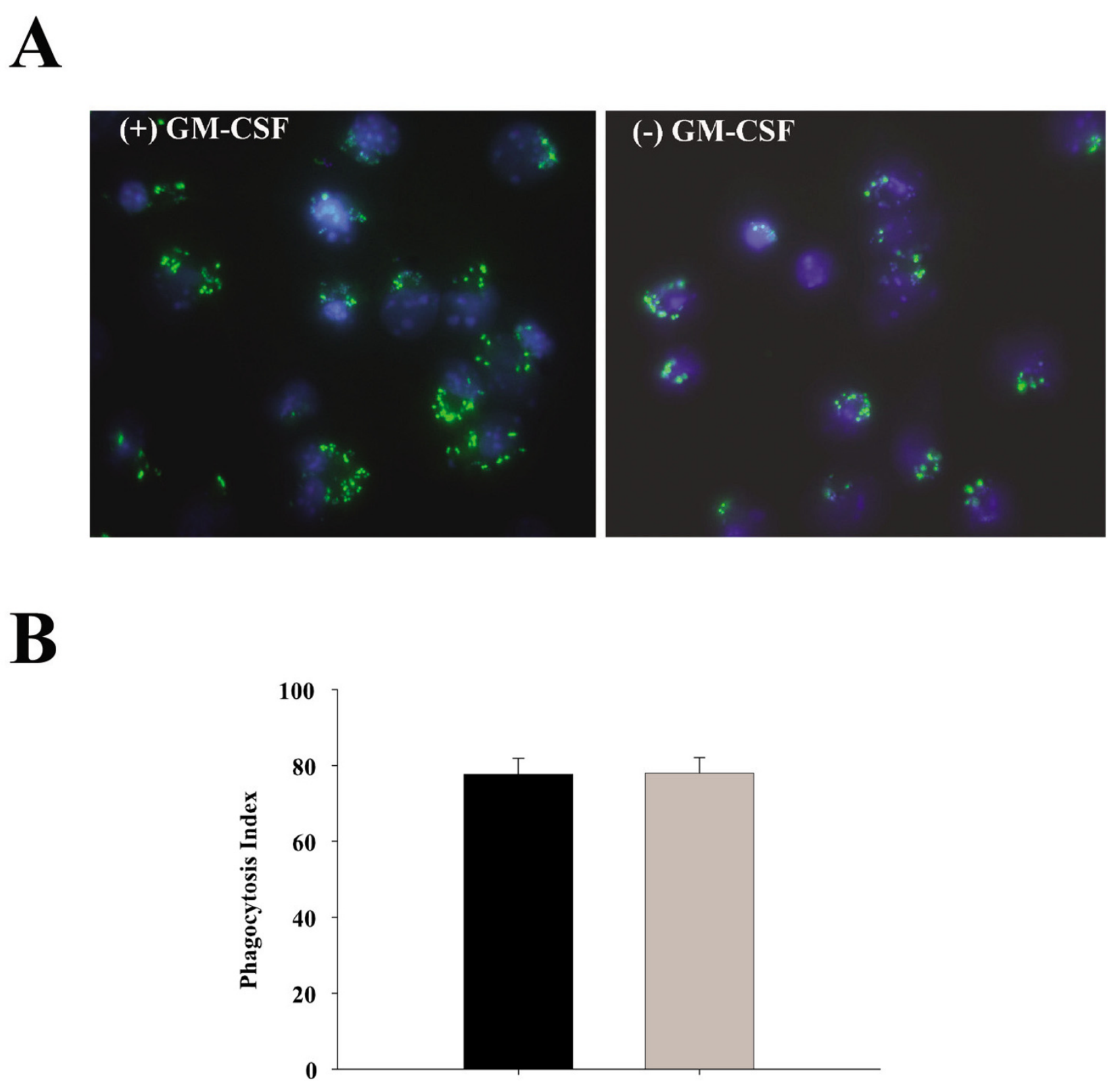

(+) GM-CSF

(-) GM-CSF

\section{Figure 5}

Phagocytic activity of primary microglia is not affected by GM-CSF. Primary microglia expanded either with (+) or without (-) GM-CSF were seeded onto $12 \mathrm{~mm}$ coverslips at $2 \times 10^{5}$ cells per coverslip and incubated overnight in 24-well plates. The following day, cells were treated with $2 \times 10^{6}$ heat-inactivated S. aureus-GFP (green) for $3 \mathrm{~h}$ and visualization of intracellular bacteria was detected using fluorescence microscopy (40x). Hoechst dye (blue) was used to visualize nuclei (A). In (B), the phagocytic index was calculated as the percentage of microglia which engulfed bacteria in a total of ten, $40 \times$ microscopic fields. The results represent the mean \pm SEM of two independent experiments.

ways. In the first, GM-CSF was provided as a supplement during the initial mixed glial culture period $[10,13,14,38,40]$, whereas in another group of studies,
GM-CSF was added as a stimulus to purified microglia that had otherwise not been previously exposed to the growth factor $[6,7,16,32,37,54]$. Another variable is the 


\title{
A
}

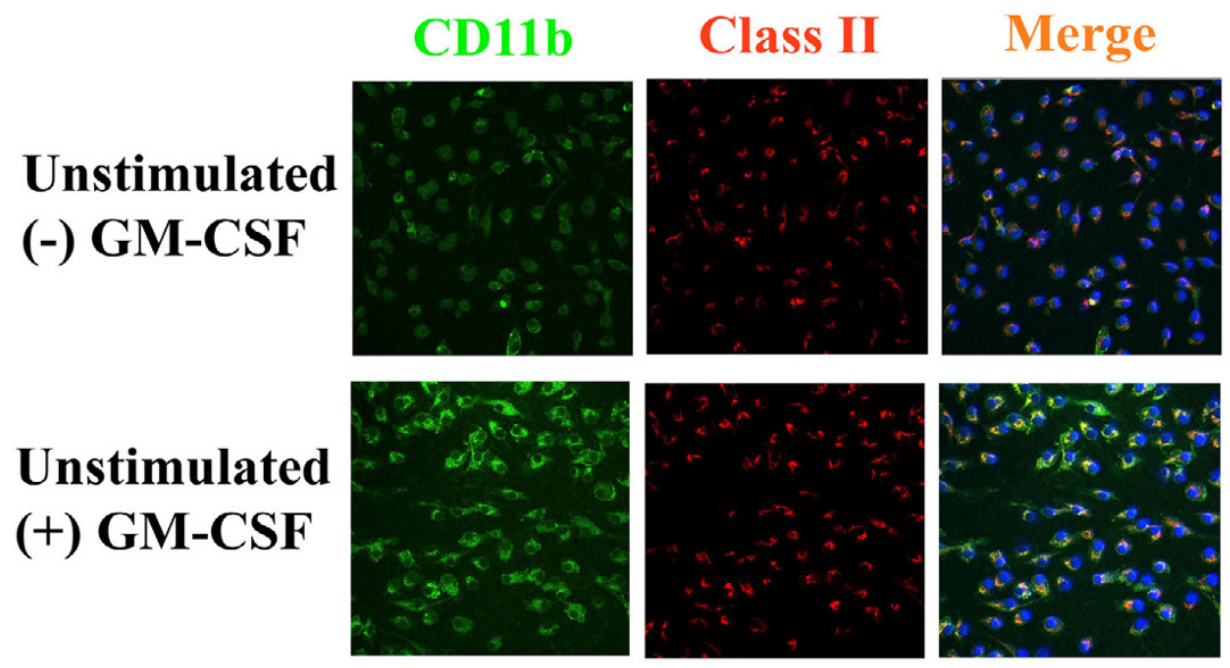

\author{
B

\section{S. aureus \\ (-) GM-CSF}

CD11b

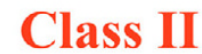

\section{Merge}
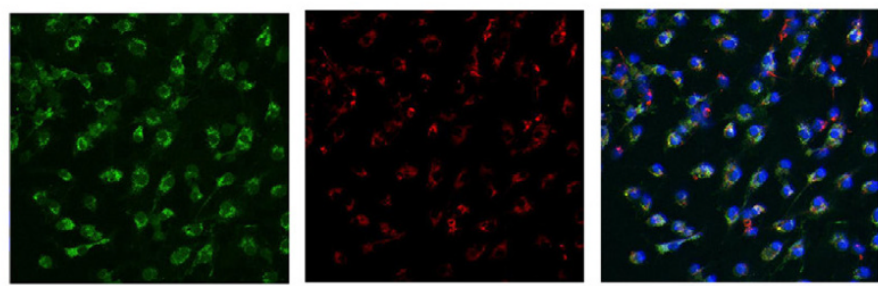

\section{S.aureus \\ (+) GM-CSF}
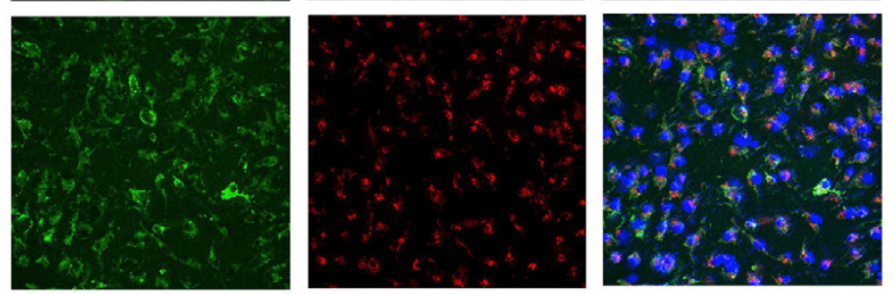

\section{C \\ Unstimulated \\ (+) GM-CSF}
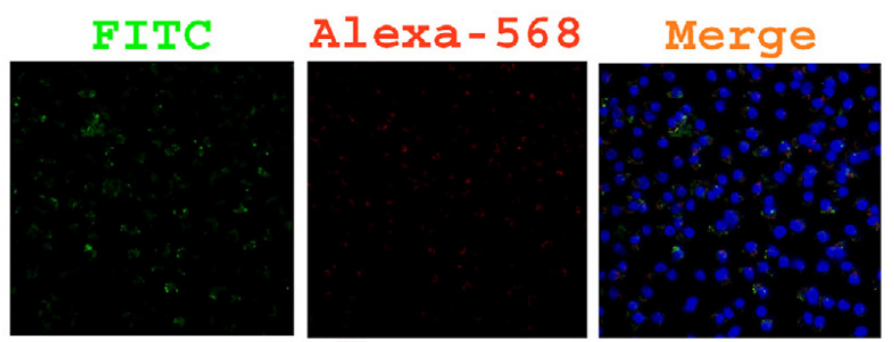

Figure 6

Microglial CD I Ib, but not MHC class II expression, is influenced by low GM-CSF levels. Primary microglia propagated either with (+) or without (-) GM-CSF were seeded onto $12 \mathrm{~mm}$ cover slips at $2 \times 10^{5}$ cells per cover slip and incubated overnight in 24-well plates. The following day, cells were either unstimulated (A) or treated with heat-inactivated S. aureus (I07 cfu/well) (B) for $24 \mathrm{~h}$, whereupon microglia were stained with CDI lb-FITC (green) and MHC Class II-Alexa-568 (red). Nuclei were visualized with Hoechst dye (blue). Panel (C) depicts background staining with secondary antibodies only. Results are representative of two independent experiments. 

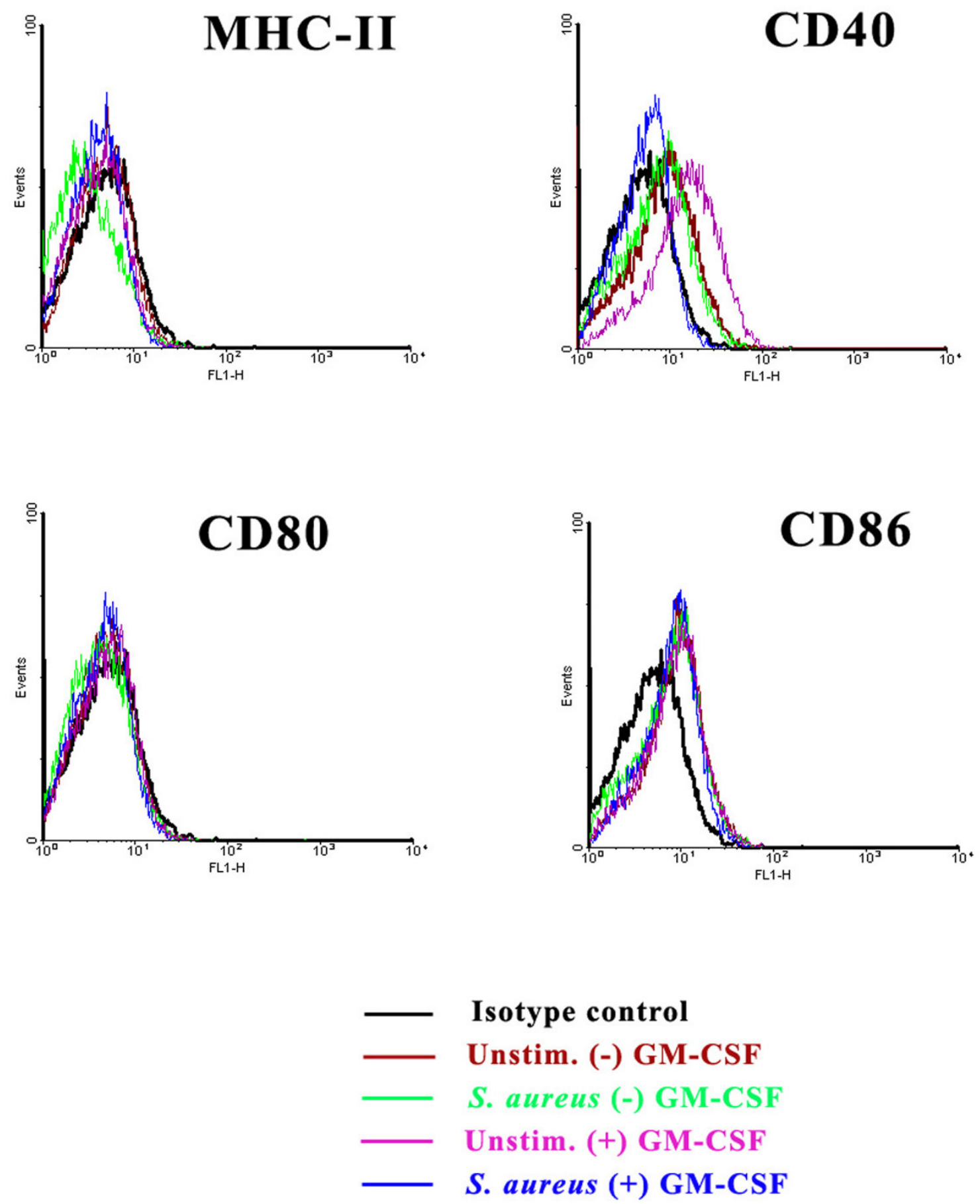

Figure 7

Low dose of GM-CSF influences microglial CD40 expression in response to S. aureus. Primary microglia expanded either with (+) or without (-) GM-CSF were seeded into 6-well plates at $2 \times 10^{6}$ cells per well and incubated overnight. The following day, cells were either unstimulated or treated with heat-inactivated S. aureus (107 cfu/well) for $24 \mathrm{~h}$, whereupon microglia were recovered and stained with MHC class II, CD40, CD80, or CD86 antibodies and subsequently with a FITC-

conjugated secondary antibody for flow cytometric analysis. Microglia were stained with an isotype-matched control antibody to assess background staining. Results are representative of two independent experiments. 

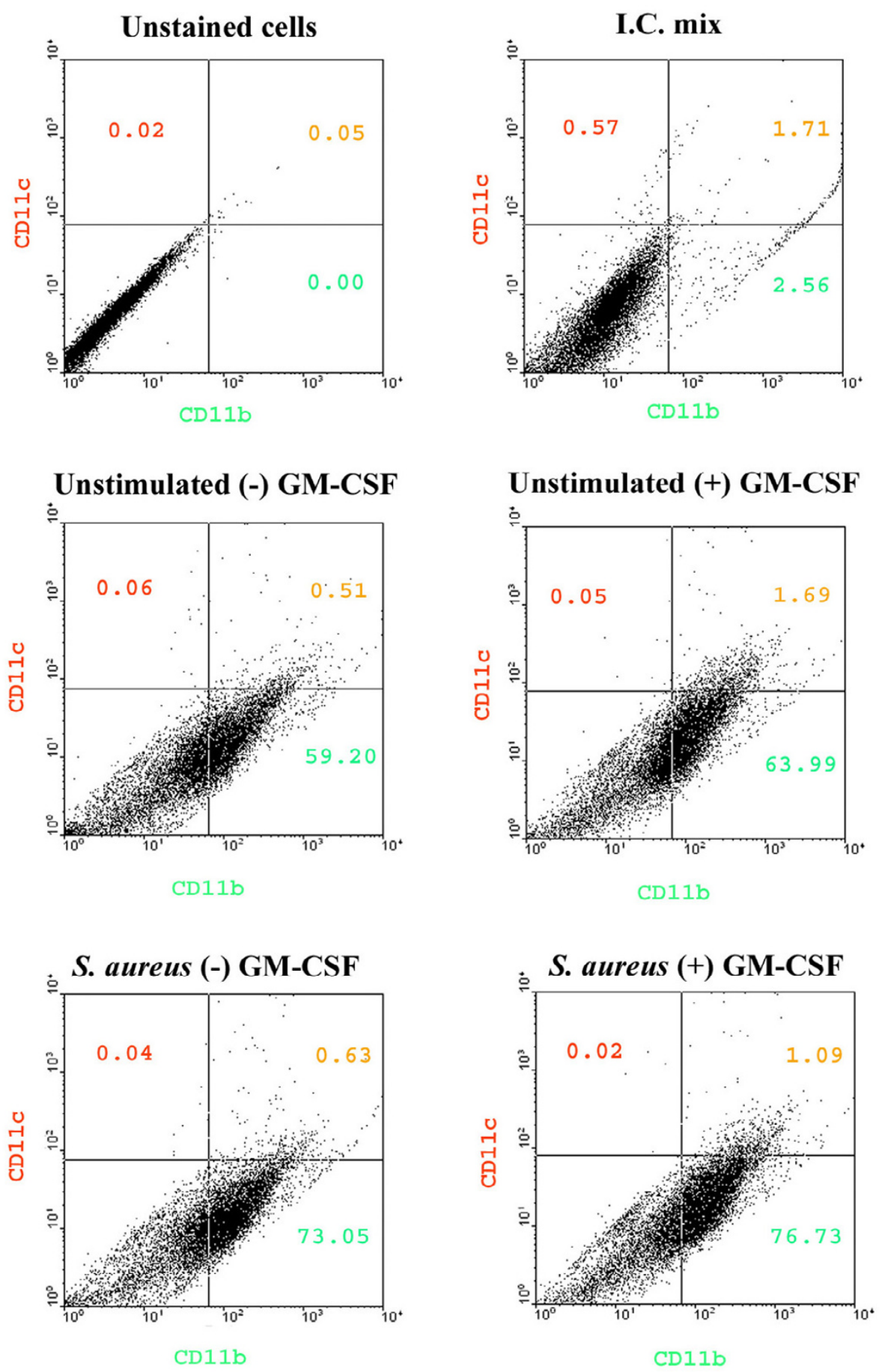

\section{Figure 8}

CDI Ic expression is not induced in neonatal microglia propagated in low dose GM-CSF. Primary microglia expanded either with (+) or without (-) GM-CSF were seeded into 6-well plates at $2 \times 10^{6}$ cells per well and incubated overnight. The following day, cells were either unstimulated or treated with heat-inactivated S. aureus ( $10^{7} \mathrm{cfu} / \mathrm{well}^{\mathrm{f}}$ ) for $24 \mathrm{~h}$, whereupon microglia were recovered and stained with CDI Ib-FITC and CDI Ic-PE-Cy7 for flow cytometric analysis. Cells were stained with isotype-matched control antibodies to demonstrate the extent of non-specific staining. The numbers shown in each quadrant represent the percentage of positive cells detected. Results are representative of two independent experiments. 
dose of GM-CSF examined which has been tested over a very broad range, namely from $0.05-1 \mu \mathrm{g} / \mathrm{ml}$ or $1-200 \mathrm{U} /$ $\mathrm{ml}$, which can be expected to have dramatic effects on the results obtained. In addition, the species from which primary microglia are procured and their maturational state, for example, mouse or rat newborn pups $[6,13,14,16,40,54]$, adult animals $[10,32,38]$, or even human fetal microglia [7] have been examined. Therefore, it is essential that the results obtained from these diverse experimental settings should be evaluated within the constraints of these parameters.

One main reason why scientists studying primary microglia have supplemented culture medium with GM-CSF is to procure sufficient numbers of microglia for downstream analysis, since GM-CSF has been shown to induce microglial proliferation $[6,10]$. Indeed, this modification has resulted in accelerated cell growth and increased cell yields, saving time and animal utilization. In our in vitro studies, we typically isolate primary mixed glial cells from newborn mice and culture them in medium supplemented with a relatively low dose of GM-CSF $(0.5 \mathrm{ng} / \mathrm{ml})$. Importantly, upon recovery of microglia from these cultures, cells are never exposed to exogenous GM-CSF again and are not utilized in experiments for at least 2 days following low dose GM-CSF withdrawal. With this modification, we were able to collect sufficient numbers of microglia for our experiments even after the third harvest, effectively reducing the need for additional microglia cultures. In addition, the main inflammatory responses of microglia following $S$. aureus stimulation, namely proinflammatory mediator production and phagocytosis, were not affected by propagating cells in the presence of GMCSF (Figures 2 and 3). Interestingly, exposure of microglia to GM-CSF modestly influenced proinflammatory mediator production following LPS stimulation, whereas responses to polyI:C were not affected (Figure 4). It has been well established that both of these PAMPs activate microglia via TLR4 and TLR3, respectively, leading to GMCSF production [62-66], as well as the priming ability of GM-CSF to augment LPS-induced TNF- $\alpha$ production in monocytes [67] and alveolar macrophages [68]. However, to the best of our knowledge, the current study represents the first report investigating the effects of low dose GMCSF on microglial responses to TLR4 and TLR3 ligands. Importantly, based on the finding that low dose GM-CSF does not dramatically alter microglial proinflammatory mediator expression in response to a diverse array of PAMPs, we propose that our cell culture procedure may be applicable to a wide range of studies investigating the immune properties of primary mouse microglia without concerns of drastically transforming cellular responsiveness. However, a word of caution must be mentioned, in that not all PAMP recognition is resilient to low dose GM-
CSF, as demonstrated by our CpG-ODN findings discussed below.

Several researchers have reported that microglia express TLR9 and respond to the TLR9 agonist CpG-ODN with the robust production of numerous proinflammatory mediators as well as increased expression of immune receptors [12,69-71]. Moreover, recent evidence demonstrating that $\mathrm{CPG}-\mathrm{ODN}$ stimulated microglia induce neuron cell death in a co-culture paradigm has suggested a link between TLRs and neurotoxicity and neurodegeneration [69]. On the other hand, we have reported that alternative TLR agonists such as $S$. aureus, PGN and LPS lead to a time-dependent decrease in TLR9 mRNA expression [20], which might be explained as a compensatory mechanism for microglia to downregulate cellular activation pathways. A recent study has shown that GM-CSF $(20 \mathrm{ng} /$ $\mathrm{ml}$ ) in combination with either CpG- or non-CpG-ODN was capable of inducing several chemokines in primary human monocytes [72], as well as in lymphoma and neuroblastoma models $[73,74]$. Although the methods used in these studies are quite distinct compared to our experimental design, they still support our findings that GMCSF exacerbates microglial activation following CpGODN stimulation (Figure 4). Our results highlight the importance of experimental culture conditions and the potential effects of GM-CSF, even at very low doses, should be considered while investigating the consequences of GpG-ODN on microglial activation. The mechanism(s) responsible for this observed synergistic effect between GM-CSF and CpG-ODN remain to be clarified with further studies. Importantly, in this study microglia expanded in the presence/absence of GM-CSF expressed equivalent levels of TLR9 mRNA under resting conditions (data not shown), suggesting that the impaired responsiveness of GM-CSF (-) microglia to subsequent ODN stimulation is likely not the result of reduced TLR9 expression.

One of the main concerns reported with GM-CSF treatment of primary microglia cultures is its ability to induce microglial differentiation towards a DC phenotype $[10,15,40]$. GM-CSF can be produced by glial cells following inflammatory or infectious diseases of the brain [7578]; therefore, it was postulated that GM-CSF may modulate the generation and maturation of DCs from microglia in the context of CNS disease [38]. Although our experiments demonstrate that low dose GM-CSF does not lead to overt alterations in the functional properties of microglia to diverse bacterial stimuli, cytokine treatment does induce morphological changes characteristic of DC-like cells. Specifically, we found that unstimulated microglia expanded in the presence of GM-CSF displayed an increased number of dendritic processes and were enlarged and more adherent compared to cells that had 
not been exposed to exogenous growth factor. Despite these phenotypic differences, we were not able to uncover any functional implications for these changes and no CD11c induction on GM-CSF exposed microglia could be demonstrated. Therefore, additional studies are warranted to further investigate the consequences, if any, of this morphological transformation of microglia following low dose GM-CSF exposure. A general consensus that can be inferred from the literature, and is in partial agreement with the results presented here, is that GM-CSF can lead to the transition of microglia into a DC-like phenotype, although the magnitude of these changes appears to be influenced by the dose of GM-CSF cells are exposed to.

The expression of other surface molecules including MHC class II, CD80, and CD86 were not affected by exposure of microglia to low dose GM-CSF. The expression of these molecules has been shown by others to be elevated in neonatal microglia, where cells exhibit a partially activated phenotype in culture $[15,52,55]$. In ex vivo isolated adult microglia, GM-CSF stimulation did not alter the expression of MHC class II, CD40, or CD86 but did induce morphological changes $[15,54]$. In contrast to our previous report demonstrating that $S$. aureus stimulation induced MHC class II and co-stimulatory molecule expression in the N9 microglial cell line [11], we found in the present study that the levels of MHC class II, CD80, and CD86 were not augmented in primary microglia following S. aureus stimulation. However, CD40 expression was dramatically upregulated following $S$. aureus stimulation in microglia that were expanded in the presence of GM-CSF. We propose that one explanation for these contradictory findings may lie in the nature of the cell types examined. For example, primary neonatal microglia in culture may inherently express higher constitutive levels of these molecules such that further activation will not result in an obvious increase in their expression. Alternatively, the length of time required to detect activationdependent increases in surface marker expression in primary microglia may be longer than that observed for the N9 cell line; however, this possibility seems less likely. Another potential explanation is that the concentration of $S$. aureus required to increase MHC class II and co-stimulatory molecule expression may differ between primary microglia and the N9 cell line. Since we only examined one dose of $S$. aureus in the present study it remains possible that titration of higher bacterial concentrations may have elicited an increase in surface marker expression, although this possibility remains speculative. The finding that MHC class II levels did not increase in S. aureus stimulated microglia when assayed using two independent approaches (i.e. immunofluorescence and flow cytometry staining) provides additional support to establish the validity of our findings.
Another important factor to consider is the nature of how primary microglia are propagated during in vitro culture. Specifically, in our studies, microglia and astrocytes are maintained as mixed cultures during the expansion period. Therefore, it is important to acknowledge that the coordinated effects of endogenous astrocyte-derived factors in combination with exogenous GM-CSF may influence the properties of recovered microglia. As an extension, it is not surprising that different microglial phenotypes are observed when cells are directly purified from the brain parenchyma and cultured in isolation. In addition, compounded with the addition of high levels of exogenous GM-CSF used in other studies, it is not unexpected that microglia assume different biological and functional properties. We propose that the maintenance of primary microglia initially as mixed cultures with astrocytes is more reminiscent of the natural milieu these cells are exposed to in vivo and represents an acceptable model to study the immunological properties of microglia, although we clearly recognize the potential for any in vitro findings to not directly equate to in vivo responses due to the artificial nature of the former. However, the examination of microglia in isolation provides a more simplified approach to investigate the effector functions of these CNS phagocytes in the absence of confounding effects by surrounding cell types.

\section{Conclusion}

Our findings demonstrate that supplementation of neonatal mouse mixed glial cultures with low dose GM-CSF successfully maintained microglial expansion and did not lead to overt alterations in the functional responses of microglia following stimulation with several PAMPs of importance during CNS bacterial and viral infections including PGN, LPS, polyI:C, as well as $S$. aureus. This suggests that our culture paradigm may be successfully used as a method to procure larger quantities of microglia without significantly affecting their downstream responses to microbial stimuli. However, it is important to note that differences in CpG-ODN responsiveness as well as the transition towards a DC-like phenotype were observed in microglia propagated in the presence of low dose GMCSF, the functional implications of which are currently unknown.

\section{Abbreviations}

APC (antigen presenting cell); CD (cluster of Differentiation); CFU (colony-forming units); CNS (central nervous system); DC (dendritic cell); DMEM (Dulbecco's modified eagle medium); ELISA (enzyme linked immunosorbent assay); FACS (fluorescence activated cell sorting); FBS (fetal bovine serum); FITC (fluorescein isothiocyanate); GFP (green fluorescence protein); IFN- $\gamma$ (interferon gamma); IL-12 p40 (interleukin-12 p40); GM-CSF (granulocyte-macrophage colony-stimulating factor); LPS 
(lipopolysaccharide); M-CSF (macrophage colony-stimulating factor); MHC (major histocompatibility complex); MIP-2/CXCL2 (macrophage inflammatory protein-2); MTT (3- [4,5-dimethylthiazol-2-yl]-2,5-diphenyl-tetrazolium bromide); ODN (oligonucleotide); OPI (oxalacetic acid, pyruvate, insulin); PAMP (pathogen associated molecular pattern); PGN (peptidoglycan); PBS (phosphate buffered saline); polyI:C (polyinosine-polycytidylic acid);S. aureus (Staphylococcus aureus); TLR (Toll-like receptor); TNF- $\alpha$ (tumor necrosis factor-alpha).

\section{Competing interests}

The author(s) declare that they have no competing interests.

\section{Authors' contributions}

Both NE and TK directed the work and prepared the manuscript.

\section{Acknowledgements}

The authors would like to thank Thuang Shwe for preparation of primary glial cultures, Dr. Mohsin Md. Syed for assistance with phase-contrast and confocal microscopy, Patrick Mayes for excellent technical assistance, and Dr. Paul Drew for critical review of the manuscript. This work was supported by the NIH National Institutes of Mental Health (ROI MH65297) and Neurological Disorders and Stroke (NINDS, ROI NS40730) to T.K. and the NINDS supported Core facility at UAMS (P30 NS047546). Support for the Digital and Confocal Microscopy Laboratory at the University of Arkansas for Medical Sciences is provided by NIH/INBRE P20 RR6460 and NIH/NCRR SIO RRI 9395.

\section{References}

I. Abbas AK, Lichtman AH: Cellular and Molecular Immunology. Philadelphia, WB Saunders; 2000:268-269.

2. Metcalf $D$ : Control of granulocytes and macrophages: molecular, cellular, and clinical aspects. Science 1991, 254:529-533.

3. Groopman JE, Molina JM, Scadden DT: Hematopoietic growth factors. Biology and clinical applications. N Engl J Med 1989, 32 I: $1449-1459$.

4. Clark SC, Kamen R: The human hematopoietic colony-stimulating factors. Science 1987, 236:1229-1237.

5. Suzumura A, Sawada M, Yamamoto H, Marunouchi T: Effects of colony stimulating factors on isolated microglia in vitro. J Neuroimmunol 1990, 30: I II-I 20.

6. Giulian D, Ingeman JE: Colony-stimulating factors as promoters of ameboid microglia. J Neurosci 1988, 8:4707-47|7.

7. Lee SC, Liu W, Brosnan CF, Dickson DW: GM-CSF promotes proliferation of human fetal and adult microglia in primary cultures. Glia 1994, I 2:309-318.

8. Henze C, Hartmann A, Lescot T, Hirsch EC, Michel PP: Proliferation of microglial cells induced by I-methyl-4-phenylpyridinium in mesencephalic cultures results from an astrocytedependent mechanism: role of granulocyte macrophage colony-stimulating factor. J Neurochem 2005, 95:1069-I077.

9. Koguchi K, Nakatsuji Y, Okuno T, Sawada M, Sakoda S: Microglial cell cycle-associated proteins control microglial proliferation in vivo and in vitro and are regulated by GM-CSF and density-dependent inhibition. J Neurosci Res 2003, 74:898-905.

10. Fischer HG, Nitzgen B, Germann T, Degitz K, Daubener W, Hadding $\mathrm{U}$ : Differentiation driven by granulocyte-macrophage colony-stimulating factor endows microglia with interferongamma-independent antigen presentation function. J Neuroimmunol 1993, 42:87-95.

11. Kielian T, Mayes P, Kielian M: Characterization of microglial responses to Staphylococcus aureus: effects on cytokine, costimulatory molecule, and Toll-like receptor expression. I Neuroimmunol 2002, 130:86-99.

12. Olson JK, Miller SD: Microglia initiate central nervous system innate and adaptive immune responses through multiple TLRs. J Immunol 2004, 173:3916-3924.

13. Santambrogio L, Belyanskaya SL, Fischer FR, Cipriani B, Brosnan CF, Ricciardi-Castagnoli P, Stern LJ, Strominger JL, Riese R: Developmental plasticity of CNS microglia. Proc Natl Acad Sci U S A 200I, 98:6295-6300.

14. Re F, Belyanskaya SL, Riese RJ, Cipriani B, Fischer FR, Granucci F, Ricciardi-Castagnoli P, Brosnan C, Stern LJ, Strominger JL, Santambrogio L: Granulocyte-macrophage colony-stimulating factor induces an expression program in neonatal microglia that primes them for antigen presentation. J Immunol 2002, 169:2264-2273.

15. Ponomarev ED, Novikova M, Maresz K, Shriver LP, Dittel BN: Development of a culture system that supports adult microglial cell proliferation and maintenance in the resting state. J Immunol Methods 2005, 300:32-46.

16. Magnus T, Korn T, Jung S: Chronically stimulated microglial cells do no longer alter their immune functions in response to the phagocytosis of apoptotic cells. J Neuroimmunol 2004, 155:64-72.

17. Delgado M, Chorny A, Ganea D, Gonzalez-Rey E: Vasoactive intestinal polypeptide induces regulatory dendritic cells that prevent acute graft versus host disease and leukemia relapse after bone marrow transplantation. Ann N Y Acad Sci 2006, 1070:226-232.

18. Vassiliou E, Jing H, Ganea D: Prostaglandin E2 inhibits TNF production in murine bone marrow-derived dendritic cells. Cell Immunol 2003, 223:120-132.

19. Esen N, Tanga FY, DeLeo JA, Kielian T: Toll-like receptor 2 (TLR2) mediates astrocyte activation in response to the Gram-positive bacterium Staphylococcus aureus. I Neurochem 2004, 88:746-758.

20. Esen N, Kielian T: Central role for MyD88 in the responses of microglia to pathogen-associated molecular patterns. J Immunol 2006, I76:6802-68II.

21. Kielian T, Haney A, Mayes PM, Garg S, Esen N: Toll-like receptor 2 modulates the proinflammatory milieu in Staphylococcus aureus-induced brain abscess. Infect Immun 2005, 73:7428-7435.

22. Kielian T, McMahon M, Bearden ED, Baldwin AC, Drew PD, Esen N: S. aureus-dependent microglial activation is selectively attenuated by the cyclopentenone prostaglandin I5-deoxyDelta | 2, I 4- prostaglandin J2 (I5d-PGJ2). J Neurochem 2004, 90:1163-1172.

23. Kielian T, Barry B, Hickey WF: CXC chemokine receptor-2 ligands are required for neutrophil-mediated host defense in experimental brain abscesses. J Immunol 200 I, 166:4634-4643.

24. Giulian D, Baker TJ: Characterization of ameboid microglia isolated from developing mammalian brain. J Neurosci 1986, 6:2163-2I78.

25. Matsumoto $Y$, Ikuta F: Appearance and distribution of fetal brain macrophages in mice. Immunohistochemical study with a monoclonal antibody. Cell Tissue Res 1985, 239:27I-278.

26. del Rio-Hortega P: Microglia. In Cytology and Cellular Pathology of the Nervous System Edited by: Penfield W. New York, Paul B. Hoeber Inc.; 1932:48I-584.

27. Giulian D: Ameboid microglia as effectors of inflammation in the central nervous system. J Neurosci Res 1987, I8: I55-71, I323 .

28. Rosenstiel P, Lucius R, Deuschl G, Sievers J, Wilms H: From theory to therapy: implications from an in vitro model of ramified microglia. Microsc Res Tech 200I, 54:18-25.

29. Shaked I, Porat Z, Gersner R, Kipnis J, Schwartz M: Early activation of microglia as antigen-presenting cells correlates with $T$ cell-mediated protection and repair of the injured central nervous system. I Neuroimmunol 2004, 1 46:84-93.

30. Aloisi F, Serafini B, Adorini L: Glia-T cell dialogue. J Neuroimmunol 2000, 107: III-II7.

31. Aloisi F, Ria F, Adorini L: Regulation of T-cell responses by CNS antigen-presenting cells: different roles for microglia and astrocytes. Immunol Today 2000, 21:141-147.

32. Aloisi F, De Simone R, Columba-Cabezas S, Penna G, Adorini L: Functional maturation of adult mouse resting microglia into an APC is promoted by granulocyte-macrophage colony- 
stimulating factor and interaction with Th I cells. J Immunol 2000, 164: $1705-17 / 2$

33. Fujita H, Tanaka J, Toku K, Tateishi N, Suzuki Y, Matsuda S, Sakanaka $M$, Maeda N: Effects of GM-CSF and ordinary supplements on the ramification of microglia in culture: a morphometrical study. Glia 1996, 18:269-28I.

34. Streit WJ: Microglia and macrophages in the developing CNS. Neurotoxicology 200I, 22:619-624.

35. Streit WJ, Walter SA, Pennell NA: Reactive microgliosis. Prog Neurobiol 1999, 57:563-58|

36. Streit W]: Microglial senescence: does the brain's immune system have an expiration date? Trends Neurosci 2006, 29:506-5I0.

37. Tambuyzer BR, Nouwen EJ: Inhibition of microglia multinucleated giant cell formation and induction of differentiation by GM-CSF using a porcine in vitro model. Cytokine 2005, 3I:270-279.

38. Fischer HG, Reichmann G: Brain dendritic cells and macrophages/microglia in central nervous system inflammation. J Immunol 200I, 166:2717-2726.

39. Garg S, Md Syed M, Kielian T: Staphylococcus aureus-derived peptidoglycan induces Cx43 expression and functional gap junction intercellular communication in microglia. I Neurochem 2005, 95:475-483.

40. Fischer HG, Bielinsky AK: Antigen presentation function of brain-derived dendriform cells depends on astrocyte help. Int Immunol I 999, I I: I 265- I 274.

4I. Kielian T, Esen N, Bearden ED: Toll-like receptor 2 (TLR2) is pivotal for recognition of $S$. aureus peptidoglycan but not intact bacteria by microglia. Glia 2005, 49:567-576.

42. Esen N, Kielian T: Recognition of Staphylococcus aureusderived peptidoglycan (PGN) but not intact bacteria is mediated by CDI 4 in microglia. J Neuroimmunol 2005, 170:93-104.

43. Ebert S, Gerber J, Bader S, Muhlhauser F, Brechtel K, Mitchell TJ, Nau $\mathrm{R}$ : Dose-dependent activation of microglial cells by Toll-like receptor agonists alone and in combination. J Neuroimmunol 2005, 159:87-96.

44. Zhang Z Trautmann K, Schluesener HJ.: Microglia activation in rat spinal cord by systemic injection of TLR3 and TLR7/8 agonists. J Neuroimmunol 2005, 164:154-160.

45. Jordan FL, Thomas WE: Brain macrophages: questions of origin and interrelationship. Brain Res 1988, 472:165-178.

46. Giulian D, Li J, Bartel S, Broker J, Li X, Kirkpatrick JB: Cell surface morphology identifies microglia as a distinct class of mononuclear phagocyte. J Neurosci 1995, 15:77|2-7726.

47. Sedgwick JD, Schwender S, Imrich H, Dorries R, Butcher GW, ter Meulen V: Isolation and direct characterization of resident microglial cells from the normal and inflamed central nervous system. Proc Natl Acad Sci U S A I99I, 88:7438-7442.

48. Fincher EF Johannsen L, Kapas L, Takahashi S, Krueger JM.: Microglia digest Staphylococcus aureus into low molecular weight biologically active compounds. Am J Physiol I996, 27 I:RI49-56.

49. Kaur C Too HF, Ling EA.: Phagocytosis of Escherichia coli by amoeboid microglial cells in the developing brain. Acta Neuropathol (Berl) 2004, 107:204-208.

50. Chan A, Magnus T, Gold R: Phagocytosis of apoptotic inflammatory cells by microglia and modulation by different cytokines: mechanism for removal of apoptotic cells in the inflamed nervous system. Glia 200I, 33:87-95.

5I. Guillemin G], Brew B]: Microglia, macrophages, perivascular macrophages, and pericytes: a review of function and identification. J Leukoc Biol 2004, 75:388-397.

52. Carson MJ, Reilly CR, Sutcliffe JG, Lo D: Mature microglia resemble immature antigen-presenting cells. Glia 1998, 22:72-85.

53. Ford AL, Goodsall AL, Hickey WF, Sedgwick JD: Normal adult ramified microglia separated from other central nervous system macrophages by flow cytometric sorting. Phenotypic differences defined and direct ex vivo antigen presentation to myelin basic protein-reactive CD4+ T cells compared. J Immunol 1995, 154:4309-432I.

54. Hayashi M, Dorf ME, Abromson-Leeman S: Granulocyte-macrophage colony stimulating factor inhibits class II major histocompatibility complex expression and antigen presentation by microglia. I Neuroimmunol 1993, 48:23-32.

55. Aloisi F Ria F, Columba-Cabezas S, Hess H, Penna G, Adorini L.: Relative efficiency of microglia, astrocytes, dendritic cells and $B$ cells in naive CD4+ $\mathrm{T}$ cell priming and ThI/Th2 cell restimulation. Eur J Immunol I999 Sep;29(9): 1999, 29:2705-27I4.

56. Alliot F, Lecain E, Grima B, Pessac B: Microglial progenitors with a high proliferative potential in the embryonic and adult mouse brain. Proc Natl Acad Sci U S A 199I, 88: I54I-I545.

57. Simard AR, Soulet D, Gowing G, Julien JP, Rivest S: Bone marrowderived microglia play a critical role in restricting senile plaque formation in Alzheimer's disease. Neuron 2006, 49:489-502.

58. Streit WJ, Graeber MB, Kreutzberg GW: Functional plasticity of microglia: a review. Glia 1988, I:301-307.

59. Dame JB, Christensen RD, Juul SE: The distribution of granulocyte-macrophage colony-stimulating factor and its receptor in the developing human fetus. Pediatr Res 1999, 46:358-366.

60. Wong RL, Lingenheld EG, Fitzgerald L, Clark RB: Murine T helper cell clones secrete granulocyte-macrophage colony-stimulating factor ( $\mathrm{GmCSF}$ ) by both interleukin-2-dependent and interleukin-2-independent pathways. Cell Immunol 1989, 1 23:445-455.

6I. Flugel A, Berkowicz T, Ritter T, Labeur M, Jenne DE, Li Z, Ellwart JW, Willem $M$, Lassmann $H$, Wekerle $H$ : Migratory activity and functional changes of green fluorescent effector cells before and during experimental autoimmune encephalomyelitis. Immunity 200I, I 4:547-560.

62. Krasowska-Zoladek A Banaszewska M, Kraszpulski M, Konat GW. Kinetics of inflammatory response of astrocytes induced by TLR 3 and TLR4 ligation. J Neurosci Res 2007, 85:205-2I2.

63. Park C Lee S, Cho IH, Lee HK, Kim D, Choi SY, Oh SB, Park K, Kim JS, Lee SJ.: TLR3-mediated signal induces proinflammatory cytokine and chemokine gene expression in astrocytes: differential signaling mechanisms of TLR3-induced IP-I0 and IL-8 gene expression. Glia 2006, 53:248-256.

64. Matsukura S Kokubu F, Kurokawa M, Kawaguchi M, leki K, Kuga H, Odaka M, Suzuki, S WS Takeuchi H, Kasama T, Adachi M.: Synthetic double-stranded RNA induces multiple genes related to inflammation through Toll-like receptor 3 depending on NF. kappaB and/or IRF-3 in airway epithelial cells. Clin Exp Allergy 2006, 36: 1049-1062.

65. Peck OM Williams DL, Breuel KF, Kalbfleisch JH, Fan H, Tempel GE, Teti G, Cook JA.: Differential regulation of cytokine and chemokine production in lipopolysaccharide-induced tolerance and priming. Cytokine 2004, 26:202-208.

66. Fan $\mathrm{H}$ Williams DL, Zingarelli B, Breuel KF, Teti G, Tempel GE, Spicher K, Boulay G, Birnbaumer L, Halushka PV, Cook JA.: Differential regulation of lipopolysaccharide and Gram-positive bacteria induced cytokine and chemokine production in splenocytes by Galphai proteins. Biochim Biophys Acta 2006, 1763:105I-1058.

67. Lendemans S Rani M, Selbach C, Kreuzfelder E, Schade FU, Flohe S.: GM-CSF priming of human monocytes is dependent on ERK I/2 activation. J Endotoxin Res 2006, I 2:10-20.

68. Bozinovski S Jones JE, Vlahos R, Hamilton JA, Anderson GP.: Granulocyte/macrophage-colony-stimulating factor (GM-CSF) regulates lung innate immunity to lipopolysaccharide through Akt/Erk activation of NFkappa B and AP-I in vivo. J Biol Chem 2002, 277:42808-428I4.

69. Iliev Al, Stringaris AK, Nau R, Neumann H: Neuronal injury mediated via stimulation of microglial toll-like receptor-9 (TLR9). Faseb J 2004, | 8:4|2-4|4

70. Dalpke AH, Schafer MK, Frey M, Zimmermann S, Tebbe J, Weihe E, Heeg K: Immunostimulatory CpG-DNA activates murine microglia. J Immunol 2002, 168:4854-4863.

7I. Takeshita S, Takeshita F, Haddad DE, Janabi N, Klinman DM, Dalpke $\mathrm{AH}$, Schafer MK, Frey M, Zimmermann S, Tebbe J, Weihe E, Heeg K, Iliev Al, Stringaris AK, Nau R, Neumann H: Activation of microglia and astrocytes by CpG oligodeoxynucleotides Immunostimulatory CpG-DNA activates murine microglia Neuronal injury mediated via stimulation of microglial toll-like receptor-9 (TLR9). Neuroreport 200I, I 2:3029-3032.

72. Wang J Alvarez R, Roderiquez G, Guan E, Caldwell Q, Wang J, Phelan $M$, Norcross MA.: CpG-independent synergistic induction of beta-chemokines and a dendritic cell phenotype by orthophosphorothioate oligodeoxynucleotides and granulocytemacrophage colony-stimulating factor in elutriated human primary monocytes. J Immunol 2005, 174:6 | |3-6I2I. 
73. Sandler AD Hiroshi Chihara, Gen Kobayashi, Xiaoyan Zhu, Michal A. Miller, David L. Scott and Arthur M. Krieg: CpG Oligonucleotides Enhance the Tumor Antigen-specific Immune Response of a Granulocyte Macrophage Colony-stimulating Factor-based Vaccine Strategy in Neuroblastoma. Cancer Research 2003, 63:394-399,.

74. Liu HM Sally E. Newbrough, Sudershan K. Bhatia, Christopher E. Dahle, Arthur M. Krieg, and George J. Weiner: Immunostimulatory CpG Oligodeoxynucleotides Enhance the Immune Response to Vaccine Strategies Involving Granulocyte-Macrophage Colony-Stimulating Factor. Blood 1998, 92:3730-3736.

75. Hunter CA, Roberts CW, Alexander J: Kinetics of cytokine mRNA production in the brains of mice with progressive toxoplasmic encephalitis. Eur J Immunol 1992, 22:2317-2322.

76. Malipiero UV, Frei K, Fontana A: Production of hemopoietic colony-stimulating factors by astrocytes. I Immunol 1990, 144:3816-382I.

77. Fischer HG, Nitzgen B, Reichmann G, Hadding U: Cytokine responses induced by Toxoplasma gondii in astrocytes and microglial cells. Eur J Immunol 1997, 27:1539-1548.

78. Lyons JA, Zhao ML, Fritz RB: Pathogenesis of acute passive murine encephalomyelitis II. Th I phenotype of the inducing population is not sufficient to cause disease. I Neuroimmunol 1999, 93:26-36.

Publish with Bio Med Central and every scientist can read your work free of charge

"BioMed Central will be the most significant development for disseminating the results of biomedical research in our lifetime. "

Sir Paul Nurse, Cancer Research UK

Your research papers will be:

- available free of charge to the entire biomedical community

- peer reviewed and published immediately upon acceptance

- cited in PubMed and archived on PubMed Central

- yours - you keep the copyright

Submit your manuscript here:

http://www.biomedcentral.com/info/publishing_adv.asp
BiolMedcentral 